Article

\title{
Ultrafast Optical Heating Induced Polarization-Dependent Optical Switching in Gold Nanowires
}

\author{
Yimeng Wang, Xinping Zhang * and Xiaohui Fang \\ Institute of Information Photonics Technology and College of Applied Sciences, Beijing University of \\ Technology, Beijing 100124, China; wangyimeng@emails.bjut.edu.cn (Y.W.); fangxh@bjut.edu.cn (X.F.) \\ * Correspondence: zhangxinping@bjut.edu.cn; Tel.: +86-10-6739-6371 \\ Academic Editors: Antonella Bogoni and Luca Poletto \\ Received: 9 October 2016; Accepted: 27 December 2016; Published: 12 January 2017
}

\begin{abstract}
Excitation using femtosecond laser pulses induced ultrafast heating of discontinuous gold nanowires, resulting in transient thermal expansion of the gold nanostructures that constitute the nanowires. The cross-plasmon resulting from the closely arranged gold nanostructures along the nanowires was modified by the change in the small gaps due to the thermal effect. This led to the spectral shift of the cross-plasmon resonance and laid the photophysical basis for the optical switching. A femtosecond pump-probe scheme was used to investigate the ultrafast optical switching dynamics. The most efficient optical switching effect was observed when the pump and probe laser pulses were polarized perpendicular and parallel to the discontinuous gold nanowires, respectively.
\end{abstract}

Keywords: ultrafast optical heating; optical switch; gold nanowires; plasmonic hot electrons; thermal expansion

\section{Introduction}

Plasmonic optical switching effects have been reported on extensively, and are based on the spectral shift of the localized surface plasmon resonance (LSPR) of the metallic nanostructures [1-4]. Modification on the electron-electron and electron-phonon scattering processes by the intensive ultrashort laser pulses has been the main physical mechanism [5-7]. Additionally, ultrafast optical switches have been proposed using nonlinear plasmonic nanoantennas [8]. Using different materials [9] and different shapes [10] of plasmonic nanostructures has diversified and enriched the research work on ultrafast plasmonic optical switching effects. Working at low optical excitation powers or excitation intensities is always a direction to strive for optical switching devices [11]. However, the amplitude of the switching signal is generally very small due to the very small laser-excitation-induced spectral shift. Therefore, amplification mechanisms are required to improve the performance of the optical switching device. For example, Fano coupling between the plasmonic and photonic resonance modes can be utilized to introduce dramatic change in the spectroscopic response, which favors enlargement of the differential spectrum that determines the amplitude of the optical switching signal $[12,13]$. Recently, a cross-plasmon has been demonstrated in the three-dimensional (3D) network of gold nanowires, where Fano coupling between Rayleigh anomaly and such cross-plasmon provides us new opportunities to achieve efficient optical switching devices [14]. In fact, such cross-plasmons can be achieved very commonly in metallic nanostructures with very small separation gaps, which depend strongly on the gap width and on the polarization direction of the light. In this work, we investigate the polarization-dependent optical switching effects in a waveguide-gold-nanowire grating. We used the thermal effects induced by femtosecond laser pulses to modify a cross-plasmon in the discontinuous gold nanowires. The thermal expansion of the gold nanostructures and the scattering of the hot plasmonic electrons have been responsible for such optical switching effects. 


\section{Fabrication and Characterization of the Plasmonic Gold Nanowires}

A photoresist (PR) grating was first fabricated using interference lithography, where a He-Cd laser at $325 \mathrm{~nm}$ was used as the ultraviolet (UV) light source and S1805 photoresist (Rohm \& Haas, Esslingen, Germany) was used as the recording medium. The PR grating with a period of about 410 $\mathrm{nm}$ was fabricated on glass substrate with a 200-nm layer of indium tin oxide (ITO), where the ITO layer also acts as a waveguide. Chemically synthesized gold nanoparticles $[15,16]$ with a diameter ranging from 5 to $10 \mathrm{~nm}$, which were suspended in xylene with a concentration of $100 \mathrm{mg} / \mathrm{mL}$, were then spin-coated onto the top surface of the grating. In the spin-coating process, a $30-\mu \mathrm{L}$ colloidal solution of gold was spin-coated at a speed of $2000 \mathrm{rpm}$. An annealing process at $280{ }^{\circ} \mathrm{C}$ enabled sublimation of the ligands on the surface of the colloidal gold nanoparticles and melting of the gold nanoparticles to be confined into the grating grooves. After being cooled down to room temperature, gold nanowires form in the grating grooves.

Figure 1a shows the scanning electron microscopic (SEM) image of the gold nanowires. Clearly, each of the gold nanowires is not continuous and smooth along the grating lines; instead, it is composed of short segments or large gold particles with small gaps in between, which results from the annealing process. However, this provides us an opportunity to adjust the gaps between these gold nanostructures using optical heating process. According to Figure 1a, there is a large number of such gaps that are smaller than $20 \mathrm{~nm}$, as indicated by the yellow arrows. Such small gaps are easily influenced by interaction between the gold nanowires and intense femtosecond laser pulses. Consequently, the localized surface plasmons resonant at these gaps may be modified by optical pulses. This is the basic mechanism for the polarization-dependent optical switching effects described in this work.
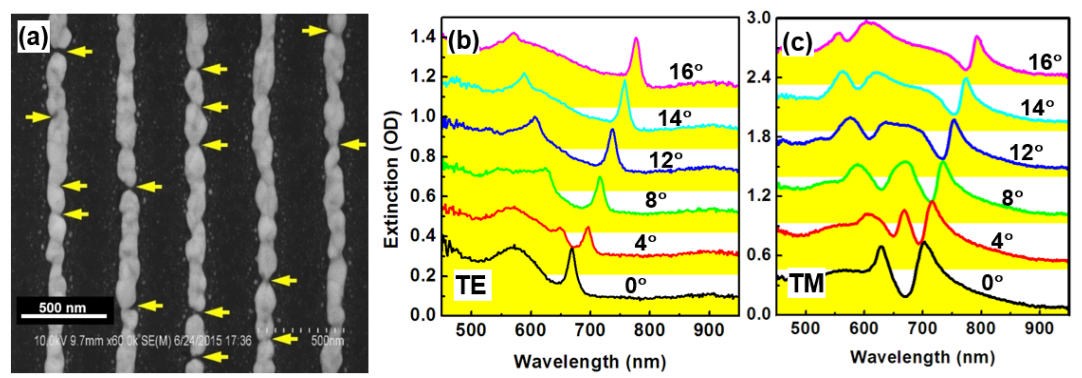

Figure 1. (a) SEM image of the gold nanowires; (b,c) optical extinction spectra measured at different angles of incidence for transverse electric (TE) and transverse magnetic (TM) polarizations, respectively.

Figure $1 b, c$ shows the optical extinction spectra measured on the gold-nanowire grating with the incident angle changed from 0 to 16 degrees for transverse electric (TE) and transverse magnetic (TM) polarizations. The light was polarized parallel and perpendicular to the gold nanowires for TE and TM polarizations, respectively. As has been investigated extensively, waveguide resonance mode will be observed for TE polarization, which exhibits narrow-band peak features in optical extinction spectra $[1,15]$. However, Fano coupling between plasmonic and waveguide resonance modes can be observed for TM polarization, as shown in Figure 1c, where enhanced transmission or narrow-band dips in the optical extinction spectrum can be observed. This Fano coupling feature provides amplification mechanisms for the optical switching process, as has been addressed in our previous publication $[1,14]$.

\section{Ultrafast Optical Switching Performance}

\subsection{Measurement Results of the Optical Switching Processes}

The optical switching effect of the gold nanowires shown in Figure 2a was investigated using femtosecond pump-probe measurements. A Ti: sapphire amplifier Legend Elite from Coherent 
provides $150 \mathrm{fs}$ pulses at $800 \mathrm{~nm}$ with a repetition rate of $1 \mathrm{kHz}$ and a maximum pulse energy of $1 \mathrm{~mJ}$. These 800-nm pulses were used as the pump after some part of the pulse energy was sent to a 1-cm cell containing $\mathrm{D}_{2} \mathrm{O}$ for supercontinuum generation. The supercontinuum pulse was used as the probe, which extends from sub-300-nm to longer than $1200 \mathrm{~nm}$ in spectrum. The delay between the pump and probe pulses was adjusted by a linear stage in the beam path of the pump pulse. The transient absorption (TA) spectrum was collected by the fiber spectrometer using CCD as the detection unit as a function of time delay.

According to Figure $1 b, c$, the optical extinction at normal incidence is about 0.22 and 0.08 OD at $800 \mathrm{~nm}$ for TM and TE polarization, respectively. Therefore, stronger interaction between the gold nanowires and the femtosecond pump laser pulses may be achieved for TM than for TE polarization. Based on this consideration, TM polarization was employed for the 800-nm pump pulses. Thus, we present experimental results for two pump-probe schemes, where the pump pulses were TM polarized and the probe were TM and TE polarized, respectively. A pump fluence of $17.8 \mu \mathrm{J} / \mathrm{cm}^{2}$ was employed in the following demonstrated experimental results. Transient absorption was evaluated by $\Delta \mathrm{A}=-\log _{10}\left(\mathrm{~T}_{\mathrm{on}} / \mathrm{T}_{\text {off }}\right)$, where $\mathrm{T}_{\text {on }}$ and $\mathrm{T}_{\text {off }}$ are the transmission rate of the probe pulse through the sample with the pump pulse switched on and off, respectively.
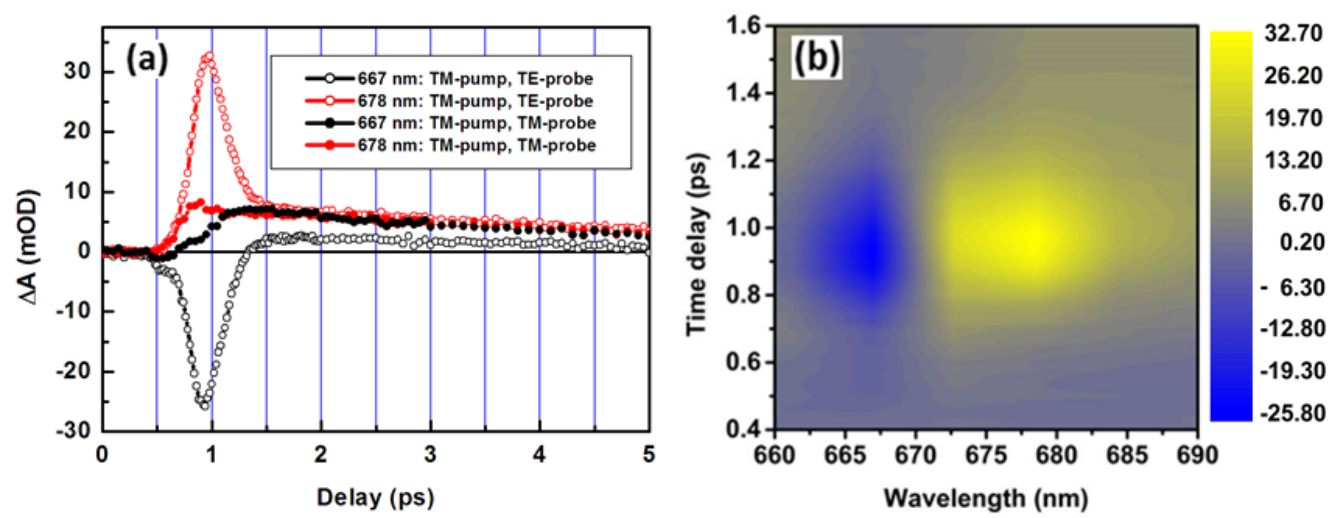

Figure 2. (a) Optical switching dynamics of the gold nanowires at two wavelengths (667 $\mathrm{nm}$ and $678 \mathrm{~nm}$ ) for two schemes of TM-pump/TM-probe and TM-pump/TE-probe; (b) 3D plot of the optical switching signal as a function of wavelength and time delay.

Figure 2a shows the pump-probe dynamics of the optical switching performance measured at two typical wavelengths of 667 (black curves) and $678 \mathrm{~nm}$ (red curves), where the open circles show TM-pump/TE-probe and the solid ones show TM-pump/TM-probe measurement results. The pump beam was sent to the sample at an angle of 2 degrees, and the probe beam was incident normal to the sample surface. For the TM-pump/TM-probe scheme, a much smaller optical switching signal with positive $\Delta \mathrm{A}$ values both at 667 and $678 \mathrm{~nm}$ were observed, as compared with that for TM-pump/TE probe. However, a strong negative signal within the first 1 ps of the time delay after the onset of the pump pulse was measured at $667 \mathrm{~nm}$, and a strong positive signal was measured at $678 \mathrm{~nm}$ for the TM-pump/TE-probe scheme. Furthermore, in TM-pump/TE-probe, a much faster optical switching process than in the TM-pump/TM-probe scheme can be observed within the first $500 \mathrm{fs}$ after the finish of the pumping process, as can be measured by evaluating the falling and rising edges of the red and black open circles, respectively, which is followed by a much slower dynamics. This faster process can be attributed to plasmonic electron-dominated scattering process, and the slower tail can be identified as a phonon-dominated process. Looking back at the two curves by solid circles, we can find that both of the optical switching signals at 667 and $678 \mathrm{~nm}$ are dominated by the slow dynamics. Comparing the optical switching dynamics of the two pump-probe schemes by the amplitude, speed, and sign of the $\Delta \mathrm{A}$ values, we can conclude that they correspond to different photophysical processes. For TE-polarized probe, we need to base our analysis on the black spectrum 
in Figure 1b, where simple waveguide resonance mode without coupling with plasmon resonance can be observed. This is because the thermal effect-induced change in the cross-plasmon can be observed only when the sample is excited by the pump pulses. In this case, 667 and $678 \mathrm{~nm}$ fall into the rising and falling edges of the spectral peak in Figure $1 \mathrm{~b}$, respectively. This is why a negative and a positive transient signal were observed at 667 and $678 \mathrm{~nm}$, respectively, due to the optical excitation-induced redshift of the spectrum. However, for TM-polarized probe, we should use the black spectrum in Figure 1c to understand the transient spectroscopic change in the Fano-coupling process. Due to the broad-band spectral feature and the location of both wavelengths at the bottom of the spectral dip, small positive transient signals were observed for TM-probing as optical excitation induced electron-electron and electron-phonon scattering, which led to the redshift of the LSPR spectrum.

Additionally, we need to note that the optical switching signal for TM-pump/TM-probe resulted from Fano coupling between the waveguide resonance mode and LSPR, while the wavelengths of 667 and $678 \mathrm{~nm}$ are located near the bottom of the coupling spectrum, as can be observed in the black spectrum in Figure 1c. The optical excitation induced redshift of LSPR and led to a complicated modulation on the spectrum of the coupled mode, which is also dependent on the pulse intensity that is varying with the pulse shape. The rising edge of the spectrum corresponds to a negative transient spectrum and falling edge to a positive value. Due to the complicated process at the bottom of the spectral dip, the dynamics may involve superimposition of pulse-intensity dependent processes. This is why dynamics at 667 and $678 \mathrm{~nm}$ for TM polarization have different rising times.

Figure $2 \mathrm{~b}$ shows a three-dimensional (3D) plot of the optical switching signal as a function of wavelength and time delay for the pump-probe scheme of TM-pump/TE-probe, which was measured at normal incidence of the probe beam. Negative TA spectra were measured at wavelength shorter than $672 \mathrm{~nm}$, and positive ones were measured at wavelengths longer than $675 \mathrm{~nm}$, which implies a red shift of the excited spectrum of plasmon resonance. Such a red shift took place within a spectral band as narrow as $20 \mathrm{~nm}$, as shown in Figure 2b. These observations enable us to find out the mechanisms for the much-enhanced optical switching signal in the TM-pump/TE-probe scheme.

\subsection{Mechanisms for the Ultrafast-Heating Enhancement}

Although the temperature dependence of dielectric permittivity of the host matrix may also contribute to the redshift of plasmon resonance [17], such mechanisms should not have polarization dependence. In this work, we observed strong redshift of the spectrum for TE polarization in the direction of the grating lines. This is the basis for us to attribute the TA spectroscopic response to a cross-plasmon at the gaps between the gold nanostructures along the grating lines. Actually, thermal expansion effects for gold nanoparticles have been investigated [18]. We made an evaluation of the thermal expansion amount of the gold nanostructures using parameters of gold: thermal expansion coefficient $\left(\alpha_{L}\right): 14 \times 10^{-6} /{ }^{\circ} \mathrm{C}$; heat capacity $\left(C_{T}\right): 128 \mathrm{~J} / \mathrm{kg} \cdot{ }^{\circ} \mathrm{C}$; and density $(\rho): 19.3 \mathrm{~g} / \mathrm{cm}^{3}$. We also use the following parameters of the gold nanostructures in our pump-probe measurements: average volume of gold nanostructures along the grating lines $(V): 3.9 \times 10^{-15} \mathrm{~cm}^{3}$; average length of the gold nano-segments $(L): 300 \mathrm{~nm}$; and estimated average optical pulse energy absorbed by such a gold nanostructure $\left(\Delta E_{O}\right): 8 \times 10^{-13} \mathrm{~J}$. Thus, if we assume homogeneous and linear thermal expansion of the gold nanostructures under optical-excitation heating, the expansion amount can be evaluated roughly by $\Delta \mathrm{L}=\alpha_{L} \cdot \frac{\Delta \mathrm{E}_{O}}{\mathrm{C}_{T} \cdot \rho \cdot V} \cdot L$. As a result, the thermal expansion of the gold nanostructures after excitation can be estimated to be $0.35 \mathrm{~nm}$.

As has been reported, a kind of cross-plasmon may be induced if the gap between the plasmonic nanostructures is smaller than $20 \mathrm{~nm}$ [19]. The resonance spectrum of such plasmons shifts to the red with reducing gap width. Figure 3 shows a series of simulation results of optical extinction spectra of the gold nanoparticles when changing the separation distance or the gap width between them. In the simulation, we used finite-difference time-domain (FDTD) method, where the parameters of the gold nanoparticles were adopted from the Palik database and the calculation was performed in the spectral range from 400 to $700 \mathrm{~nm}$. The light beam was assumed to be polarized along the 
co-axis of the two gold nanoparticles, and the gold nanoparticles were assumed to be immersed in air with an ITO glass substrate. The gold nanoparticles have the same diameter of $100 \mathrm{~nm}$. The optical extinction spectrum was calculated at different separation distances (d). As shown in Figure 3, the optical extinction spectrum shifted from about 633 to $565 \mathrm{~nm}$ when $d$ was increased from 2 to $15 \mathrm{~nm}$. Furthermore, the amplitude of the optical extinction spectrum was also reduced with increasing separation distances, corresponding to the reduction in the cross coupling between the gold nanoparticles. It can be understood that a sufficiently large separation between the gold nanoparticles corresponds to a system consisting of isolated gold nanoparticles, where the spectrum of plasmon resonance is a response of a single gold nanoparticle.

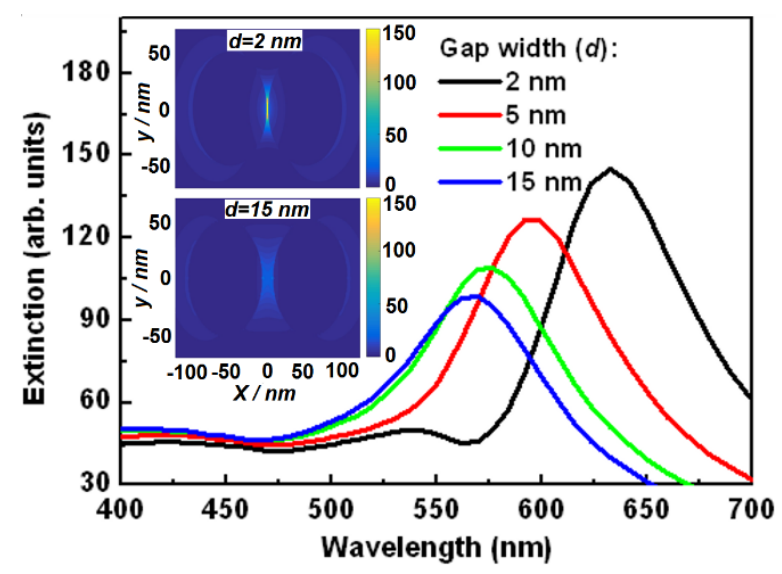

Figure 3. Calculated optical extinction spectrum at different gap width of the dimer system consisting of two identical gold nanoparticles with a diameter of $100 \mathrm{~nm}$. Inset: schematic illustration of a dimer system consisting of two gold nanoparticles with a gap width of 2 and $15 \mathrm{~nm}$, showing the calculated optical electric field distribution.

Excitation using femtosecond pulses has heated the electrons in the gold nanowires. Such a heating process led to thermal expansion of the nanostructures along the gold nanowires. However, red-shift of the resonance spectrum has verified a reduction process of the gap width between the gold nanostructures appearing as particles or segments.

\subsection{Pump Fluence Dependence of the Ultrafast Optical Switching Effect}

Figure $4 \mathrm{a}, \mathrm{b}$ shows the measurement results on the pump fluence dependence of the transient spectrum for TE and TM polarization, respectively, where the pump fluence was increased from 1.8 to $23.1 \mu \mathrm{J} / \mathrm{cm}^{2}$. To demonstrate the large difference in the optical switching effect between TE and TM polarization, we have scaled the vertical axis using the same range of $\Delta \mathrm{A}$. We can observe a much larger amplitude of the TA spectrum for TE than for TM polarization in the spectral range from 625 to $725 \mathrm{~nm}$, verifying our proposed mechanisms in this work. Narrow-band TA spectra with negative features on the left and positive on the right for TE polarization imply a red-shift of the resonance spectrum. However, for TM polarization, we observe a negative dip centered at about $690 \mathrm{~nm}$ with relatively broad-band positive spectra on both sides, indicating complicated transient processes involved in the excitation of the Fano resonance mode.

For both TE and TM polarization, we observed increased amplitude of the TA spectrum with increasing pump fluence, which is convincing evidence that higher pump fluence induced larger spectral shift of the plasmon resonance. For clearer observation of the red-shifted plasmon resonance, we rebuilt the spectra of the optically excited plasmon resonance by including the TA spectra into the steady-state optical extinction spectrum. Assuming that the optical extinction spectrum of the gold nanowires is described by $\mathrm{A}(\lambda)$ for TE polarization with $\mathrm{A}(\lambda)=-\log _{10} \mathrm{~T}(\lambda)$ and the TA spectrum by $\triangle \mathrm{A}(\lambda)$ in $m O D$, where $\mathrm{T}(\lambda)$ is the transmission spectrum through the gold nanowires, the optical 
extinction spectrum of the optically excited plasmon can be written as: $A_{E}(\lambda)=A(\lambda)+\Delta A(\lambda) / 1000$. Figure $4 \mathrm{c}$ shows the plots of $\mathrm{A}_{\mathrm{E}}(\lambda)$ at pump fluence of 1.8, 5.3, 10.7, 17.8, and $23.1 \mu \mathrm{J} / \mathrm{cm}^{2}$. $\mathrm{A}(\lambda)$ was also plotted in Figure $4 \mathrm{c}$ by the dotted curve for comparison. We can observe a red-shift of more than $2 \mathrm{~nm}$ when the pump fluence was increased from 0 to $23.1 \mu \mathrm{J} / \mathrm{cm}^{2}$. Thus, the results in Figure 4 verified different plasmonic response for different polarizations, where the scheme of TM-pump/TE-probe has much higher efficiency than TM-pump/TM-probe, and higher pump fluence resulted in larger spectral shift of the cross-plasmon for TE polarization.
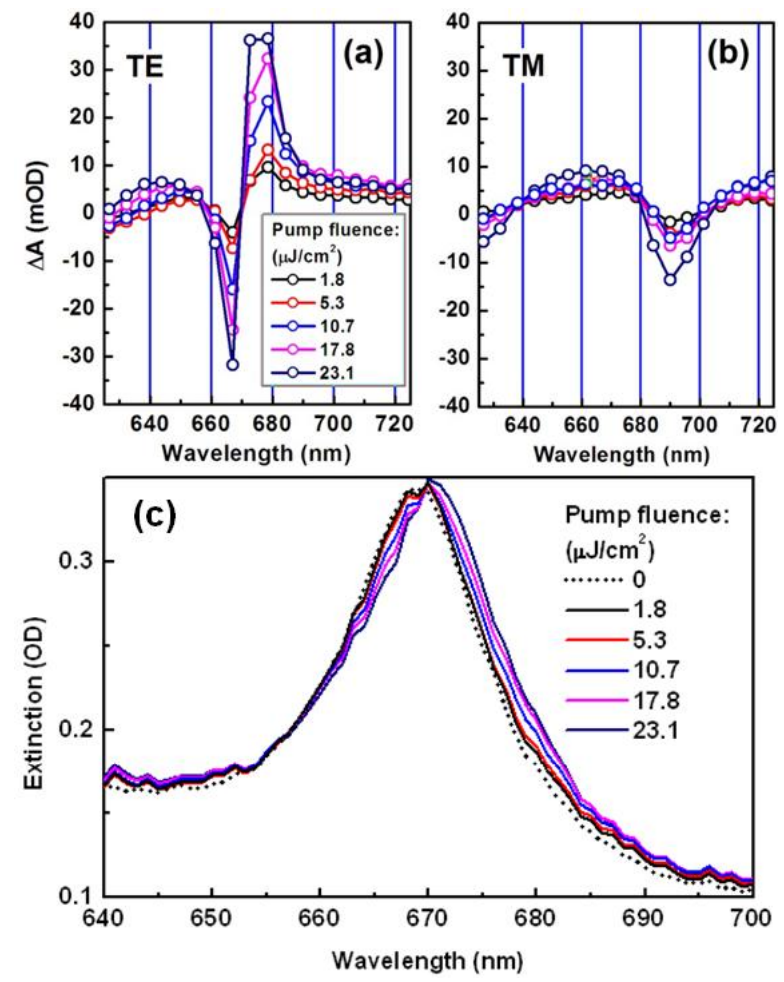

Figure 4. $(\mathbf{a}, \mathbf{b})$ Measurements on the transient spectrum for TE and TM polarization, respectively, as a function of the pump fluence increased from 1.8 to $23.1 \mu \mathrm{J} / \mathrm{cm}^{2}$; (c) Rebuilt optical extinction spectra with the transient absorption (TA) spectra included for TE polarization at different pump fluence, showing the red-shift of plasmon resonance with increasing pump fluence.

We may verify the agreement between our proposed mechanisms and the TA measurements by a comparison between the simulation results in Section 3.2 and the experimental results in Section 3.3. If we assume an approximately linear red-shift of the resonance spectrum with the reduction in the gap width, we can estimate a spectral shift of about $(633-565) /(15-2) \times 0.35 \approx 1.8 \mathrm{~nm}$, where we have used the thermal expansion amount of the gold nanostructures as large as $0.35 \mathrm{~nm}$ under optical excitation. The measured spectral shift using the rebuilt spectra in Figure $4 \mathrm{c}$ is about $2.2 \mathrm{~nm}$ at the peaks and at the falling edges. However, such a difference justifies a rough agreement between the simulation and the measurement. The reasons for the deviation may be understood by considering the deviation of the dimmer spheres in the modeling from the practical plasmonic nano-segments in the sample.

\section{Conclusions}

In conclusion, we demonstrated ultrafast optical switching processes using gold nanowires through femtosecond laser pulse induced thermal effects. Small gaps between the gold nanostructures constituting the gold nanowires-which appear as nanoparticles or small segments-induced strong cross-plasmons that are dependent on the gap widths. Such cross-plasmons were modified by 
changing the gap width through thermal expansion processes, as well as the concurrently modified electron-electron and electron-phonon scattering processes. The importance of this experimental work lies mainly in the investigation of the polarization dependence of the optical switching effect of the waveguide gold nanowire grating. Furthermore, cross-plasmon through interaction between adjacent gold nanostructures along the grating lines-instead of LSPR of each gold nanowire for polarization perpendicular to the grating lines-has been the main mechanism under strong optical pulse excitation. In particular, the efficiency of optical switching was improved remarkably in this work, where we obtained more than $7 \%$ modulation on the transmission in the optical switching signal at a pump fluence of less than $18 \mu \mathrm{J} / \mathrm{cm}^{2}$ using TM-pump/TE-probe. However, in our previous work in [1], we achieved a maximum switching signal as large as $5 \%$ modulation on the transmission at a pump fluence of about $30 \mu \mathrm{J} / \mathrm{cm}^{2}$ using TM-pump/TM-probe. The efficiency improvement is more than doubled. Such enhancement has been observed only for the light polarization along the gold nanowires.

Acknowledgments: We acknowledge the 973 Program (2013CB922404), the National Natural Science Foundation of China (11274031, 11574015), and the Beijing Key Lab of Microstructure and Property of Advanced Materials for the support.

Author Contributions: Yimeng Wang did all of the experiments and performed some of the data analysis and theoretical simulation. Xinping Zhang designed this work, performed data analysis, and wrote the paper. Xiaohui Fang helped with the theoretical simulations.

Conflicts of Interest: The authors declare no conflicts of interest.

\section{References}

1. Zhang, X.P.; Sun, B.Q.; Hodgkiss, J.M.; Friend, R.H. Tunable ultrafast optical switching via waveguided gold nanowires. Adv. Mater. 2008, 20, 4455-4459. [CrossRef]

2. Stern, L.; Grajower, M.; Levy, U. Fano resonances and all-optical switching in a resonantly coupled plasmonic-atomic system. Nat. Commun. 2014, 5, 9. [CrossRef] [PubMed]

3. Chang, W.S.; Lassiter, J.B.; Swanglap, P.; Sobhani, H.; Khatua, S.; Nordlander, P.; Halas, N.J.; Link, S. A plasmonic fano switch. Nano Lett. 2012, 12, 4977-4982. [CrossRef] [PubMed]

4. Luk'yanchuk, B.; Zheludev, N.I.; Maier, S.A.; Halas, N.J.; Nordlander, P.; Giessen, H.; Chong, C.T. The fano resonance in plasmonic nanostructures and metamaterials. Nat. Mater. 2010, 9, 707-715. [CrossRef] [PubMed]

5. Link, S.; El-Sayed, M.A. Spectral properties and relaxation dynamics of surface plasmon electronic oscillations in gold and silver nanodots and nanorods. J. Phys. Chem. B 1999, 103, 8410-8426. [CrossRef]

6. Voisin, C.; Del Fatti, N.; Christofilos, D.; Vallee, F. Ultrafast electron dynamics and optical nonlinearities in metal nanoparticles. J. Phys. Chem. B 2001, 105, 2264-2280. [CrossRef]

7. Tas, G.; Maris, H.J. Electron-diffusion in metals studied by picosecond ultrasonics. Phys. Rev. B 1994, 49, 15046-15054. [CrossRef]

8. Nicolas, L.; Martina, A.; Javier, A.; Otto, L.M. Photoconductively loaded plasmonic nanoantenna as building block for ultracompact optical switches. Nano Lett. 2010, 10, 1741-1746.

9. Hideyuki, I.; Koichiro, T.; Ichiro, T.; Toshiaki, H.; Hiroki, N. Ultrafast optical switching in a silver nanoparticle system. Jpn. J. Appl. Phys. 2000, 39, 5132-5133.

10. Yih, L.H.; Yan, Y.L.; Lakshminarayana, P.; Xua, Q.H. Nonlinear optical switching behavior of Au nanocubes and nano-octahedra investigated by femtosecond Z-scan measurements. Appl. Phys. Lett. 2009, 95, 023105.

11. Zhu, Y.; Hu, X.Y.; Huang, Y.Y.; Yang, H.; Gong, Q.H. Fast and low-power all-optical tunable Fano resonance in plasmonic microstructures. Adv. Opt. Mater. 2013, 1, 61-67.

12. Wu, J.H.; Gao, J.Y.; Xu, J.H.; Silvestri, L.; Artoni, M.; La Rocca, G.C.; Bassani, F. Ultrafast all optical switching via tunable fano interference. Phys. Rev. Lett. 2005, 95, 057401. [CrossRef] [PubMed]

13. LaGasse, M.J.; Anderson, K.K.; Haus1, H.A.; Fujimoto, J.G. Femtosecond all-optical switching in AlGaAs waveguides using a time division interferometer. Appl. Phys. Lett. 1989, 54, 2068. [CrossRef]

14. Lin, Y.H.; Zhang, X.P.; Fang, X.H.; Liang, S.Y. A Cross-stacked plasmonic nanowire network for high-contrast femtosecond optical switching. Nanoscal 2016, 8, 1421-1429. [CrossRef] [PubMed] 
15. Zhang, X.P.; Sun, B.Q.; Richard, H.F. Metallic photonic crystals based on solution-processible gold nanoparticles. Nano Lett. 2006, 6, 651-655. [CrossRef] [PubMed]

16. Kumar, S.; Pal, S.K.; Kumar, P.S.; Lakshminarayanan, V. Novel conducting nanocomposites: Synthesis of triphenylene-covered gold nanoparticles and their insertion into a columnar matrix. Soft Matter 2007, 3, 896-900. [CrossRef]

17. Yeshchenko, O.A.; Bondarchuk, I.S.; Gurin, V.S.; Dmitruk, I.M.; Kotko, A.V. Temperature dependence of the surface plasmon resonance in gold nanoparticles. Surf. Sci. 2013, 608, 275-281. [CrossRef]

18. Plech, A.; Kotaidis, V.; Lorenc, M.; Boneberg, J. Femtosecond laser near-field ablation from gold nanoparticles. Nat. Phys. 2006, 2, 44-47. [CrossRef]

19. Rechberger, W.; Hohenau, A.; Leitner, A.; Krenn, J.R.; Lamprecht, B.; Aussenegg, F.R. Optical properties of two interacting gold nanoparticles. Opt. Commun. 2003, 220, 137-141. [CrossRef]

(C) 2017 by the authors; licensee MDPI, Basel, Switzerland. This article is an open access article distributed under the terms and conditions of the Creative Commons Attribution (CC-BY) license (http://creativecommons.org/licenses/by/4.0/). 